ORIGINAL

\title{
Effect of L-glutamine levels in piglets diets challenged with Escherichia coli lipopolysacharides
}

\author{
Efecto de los niveles de L-glutamina en dietas para lechones \\ desafiados con lipopolisacáridos de \\ Escherichia coli
}

\author{
Arturo Pardo L, ${ }^{1}$ Ph.D, Angela Poveda P, ${ }^{2 *}$ Ph.D, Caio da Silva, ${ }^{1}$ Ph.D, \\ Andréa dos Santos, ${ }^{2}$ Ph.D, Emerson Venâncio, ${ }^{3}$ Ph.D, Vânia Arantes, ${ }^{4}$ Ph.D, \\ Eduardo Nogueira, ${ }^{5}$ Ph.D.
}

${ }^{1}$ Londrina State University, Animal Science Department. Rodovia Celso Garcia Cid, PR $445 \mathrm{Km}$ 380, University Campus. Londrina-Paraná, Brasil. ${ }^{2}$ Federal University of Mato Grosso, Zootechnical Department, Rondonópolis Campus, Rodovia MT-270, Parque Sagrada Familia. Rondonópolis-Mato Grosso, Brasil. ${ }^{3}$ Londrina State University, Departament of Pathological Science. Rodovia Celso Garcia

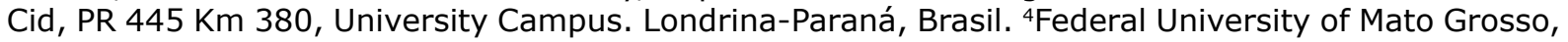
Zootechnical Department, Cuiabá Campus, Av. Fernando Corrêa da Costa, 2367. Cuiabá-Mato Grosso, Brasil. ${ }^{5}$ Ajinomoto of Brasil. Ajinomoto Animal Nutrition. Rua Joaquim Távora, 845. São Paulo-São Paulo, Brasil. *Correspondence: angelpov@gmail.com

Received: October 2013; Accepted: March 2014.

\begin{abstract}
Objective. To evaluate the effect of different levels of L-glutamine on weaned and immunologically challenged piglets with Escherichia coli lipopolysaccharides (LPS) on performance parameters, serum cortisol and defense cells. Materials and methods. Four levels of $L$-glutamine were evaluated $(0$, $1.0,1.5,2.0 \%)$ as well as the addition, or no addition, of LPS $(0.3 \mu \mathrm{g})$. 96 piglets were used (48 castrated males and 48 females) of Agroceres $\times$ PenArlan lineage, with an initial age of 21 days and $6.06 \pm 0.852 \mathrm{~kg}$ live weight. An experimental design was used on randomized blocks in a factorial setting $4 \times 2$ (levels of L- glutamine with or without challenge). Results. Cubic effect was shown for daily weight gain of unchallenged animals, and was better with the addition of $0.41 \%$ L- glutamine. Feed conversion improved with increased levels of $L$-glutamine for challenged animals. In the evaluation of defense cells, there was interaction of leukocytes with the levels of L- glutamine and the immune challenge. Eosinophils and lymphocytes showed a quadratic effect for the levels of $L$-glutamine, with a maximum value of $1.30 \%$ and $0.5 \%$, respectively. Conclusions. $L$-glutamine supplementation of up to $2 \%$ in the diet improves feed conversion and favors the immune serum of weaned piglets challenged with LPS of $E$. coli.
\end{abstract}

Key words: Animal performance, immunity, leukocytes, stress (Source: Agrovoc).

\section{RESUMEN}

Objetivo. Evaluar el efecto de diferentes niveles de L-glutamina en lechones destetados y desafiados imunológicamente con lipopolisacáridos de Escherichia coli (LPS) sobre parámetros de desempeño, cortisol sérico y células de defensa. Materiales y métodos. Se evaluaron cuatro niveles de L-glutamina 
$(0,1.0,1.5,2.0 \%)$ y la adición o no de LPS $(0.3 \mu \mathrm{g})$. Se utilizaron 96 lechones (48 machos castrados y 48 hembras) de linaje Agroceres x PenArlan, con edad inicial de 21 días y $6.06 \pm 0.852 \mathrm{~kg}$ de peso vivo. Se utilizó un diseño experimental en bloques al azar en un arreglo factorial $4 \times 2$ (niveles de L-glutamina con o sin desafío). Resultados. Se evidenció efecto cúbico para la ganancia de peso diaria para los animales no desafiados, que fue mejor con la inclusión de $0.41 \%$ de L-glutamina. La conversión alimenticia mejoró con el aumento de los niveles de L-glutamina para los animales desafiados. En la evaluación de las células de defensa hubo interacción de los leucocitos con los niveles de L-glutamina y el desafío inmunológico. Lo esosinofilos y linfocitos presentaron un efecto cuadrático para los niveles de L-glutamina siendo el valor máximo de $1.30 \%$ y $0.59 \%$, respectivamente. Conclusiones. El suplemento de L-glutamina hasta el $2 \%$ en la dieta, mejora la conversión alimenticia y favorece la inmunidad sérica de lechones destetados y desafiados con LPS de E. coli.

Palabras clave: Desempeño animal, estrés, leucocitos, inmunidad (Fuente: Agrovoc).

\section{INTRODUCTION}

Weaning in the swine industry is done between 21 and 28 days of age, at this stage psychological factors (maternal and litter segregation), social (establishment of the hierarchy in the new group) and nutritional (physical change of diet and type of food) significantly affect the development of the animals $(1,2)$.

From the dietary or nutritional point of view, interruption of breastfeeding and substitution of this source of energy/protein with plant products requires morphological, enzymatic and metabolic adaptation (3). This leads to alterations in intestinal flora, causing a reduction in the population of beneficial bacteria as a result of pathogens, which produce toxic metabolic, causing inflammation and exfoliation of the piglet's intestinal mucous membrane, generating diarrhea and underdevelopment, when death does not occur (4).

The set of alterations causes a typical picture of stress, increasing levels of corticotropin releasing factor (CRF) and serum cortisol, influencing the behavior of the animal and corresponding to an increase in the production of immune cells, which may interfere with metabolism and animal performance (5).

Various remedies have been used to minimize problems resulting from weaning. The inclusion of glutamine in the piglets' diet is one of the recent tools that favor high replication of gastrointestinal tract and immune system cells (6). Glutamine is the most abundant free amino acid in physiological fluids (plasma, cytoplasm, milk and fetal fluids), and is also present in animal and plant protein $(7,8)$. It is considered the main energy substrate for rapidly proliferating cells such as activated enterocytes and lymphocytes (9); moreover, it is essential in the synthesis of mucin and for maintaining barriers against bacterial attacks (10).

\section{INTRODUCCIÓN}

El destete en la indústria porcina es realizado entre los 21 y 28 días de edad, en esta fase factores sicológicos (segregación maternal y de su camada), sociales (establecimiento de la jerarquia en el nuevo grupo) y nutricionales (cambio físico de la dieta y tipo de alimento) afectan de manera importante el desarrollo de los animales $(1,2)$.

Desde el punto de vista alimenticio o nutricional, la interrupción de la ingesta de leche materna y la sustitución de esta fuente energética/proteica por productos de origen vegetal demandan una adaptación morfológica, enzimática y metabólica (3). Esto conlleva a alteraciones en la flora intestinal, provocando una reducción de la población de bacterias benéficas, como consecuencia de los agentes patógenos, los cuales producen metabólicos tóxicos, causando inflamación y exfoliación de la mucosa intestinal del lechón, generando cuadros diarreicos y subdesarrollo, cuando no ocurre la muerte del lechón (4).

El conjunto de alteraciones causa un cuadro típico de estrés, aumentando los niveles del factor liberador de corticotropina (CRF) y del cortisol sérico, influenciando el comportamiento del animal y coincidiendo con un aumento en la producción de células del sistema inmune, el cual puede interferir en el metabolismo y desempeño animal (5).

Diversos recursos han sido utilizados para minimizar los problemas resultantes del destete, la inclusión de glutamina a la dieta de lechones representa una de las herramientas recientes que favorecen la alta replicación de las células del tracto gastrointestinal y de las células del sistema inmune (6). La glutamina es el aminoácido libre más abundante en los fluidos fisiológicos (plasma, citoplasma, leche y fluidos fetales), asimismo está presente en la proteína animal y vegetal $(7,8)$. Es considerado el principal sustrato energético de las células de proliferación rápida como enterocitos y linfocitos activados (9); además, es esencial en la síntesis de mucina y en 
Due to the many important metabolic processes in which glutamine participates, such as nitrogen transport and donation, control, acid-base balance and in the integrity of tissues (11), research has intensified with the aim of establishing glutamine as an amino acid conditionally essential for health (12).

Administration of lipopolysaccharide (LPS) of E. coli causes a series of direct morphological alterations in the gastrointestinal tract, characterized by the decrease in height of intestinal villi and the increase of intestinal crypts (13), increased levels of pro inflammatory cytokines TNF-a and IL-6 (14), and reduced consumption.

The aim of this study was to evaluate different levels of L-glutamine in the diets of weaned piglets challenged with Escherichia coli lipopolysaccharides, on performance parameters, serum cortisol and defense cells.

\section{MATERIALS AND METHODS}

Study site and animals. The experiment was performed in the Swine Sector of the Farm School at Londrina State University (Paraná-Brazil) located at an altitude of 532 meters above sea level with coordinates between $23^{\circ} 20^{\prime} 23.45^{\prime \prime}$ South latitude and $51^{\circ} 12^{\prime} 32.28^{\prime \prime}$ West longitude; with a pre mountain humid tropical climate; with an average temperature of $20^{\circ} \mathrm{C}$, ranging from 16 to $27^{\circ} \mathrm{C}$ with annual rainfall of $1588 \mathrm{~mm}$.

96 piglets were used with Agroceres x PenArlan lineage (48 castrated males and 48 females) weaned at 21 days of age, with an average weight of $6.06 \pm 0.852 \mathrm{~kg}$, housed in 48 cages (24 metal cages with raised floors and 24 on concrete floors) with one male and one female per cage. The animals received food and water "ad libitum" during the entire experimental period.

Treatments. The experimental diets were formulated to meet recommendations described by Rostagno (15) for pigs in the pre-initial phase I (1-15 days) and initial phase II (15-30 days). The nutritional and centesimal composition is found in table 1 .

The experimental diets had different inclusions $(0,1.0,1.5$ and $2.0 \%)$ of L-glutamine (Ajinomoto Brazil) and were either associated or not associated with an immunological challenge with LPS from E. coli (serotype 0111:B4, Sigma Aldrich $^{\circledR}$ ). The challenge program with LPS was performed between 43 to 47 days after birth, where half of the animals received $0.3 \mu \mathrm{g}$ of LPS (serotype 0111:B4, Sigma Aldrich ${ }^{\circledR}$ ) diluted in 5 el mantenimiento de las barreras contra ataques de bacterias (10).

Debido a sus múltiples procesos metabólicos de gran importancia en los cuales la glutamina participa como en el transporte y donación de nitrógeno, control, equilibrio acido-base y en la integridad de tejidos (11), las investigaciones se han intensificado con el objetivo de establecer la glutamina como un aminoácido condicionalmente esencial para la salud (12).

La administración de lipopolisacáridos (LPS) de E. coli provoca una serie de alteraciones morfológicas directas en el tracto gastrointestinal, caracterizados por la disminución de la altura de las vellosidades intestinales y aumento de las criptas intestinales (13), aumentar los niveles de citocinas pro inflamatorias TNF-a y IL-6 (14), y reducir el consumo.

El objetivo de este trabajo fue evaluar diferentes niveles de L-glutamina en dietas para lechones destetados y desafiados con lipopolisacáridos de Escherichia coli, sobre parámetros de desempeño, cortisol sérico y células de defensa.

\section{MATERIALES Y MÉTODOS}

Sitio de estudio y animales. El experimento se realizó en el Sector de Porcicultura de la Fazenda Escola de la Universidade Estadual de Londrina (Paraná-Brasil) ubicada a una altitud de 532 msnm con las coordenadas entre $23^{\circ} 20^{\prime} 23.45^{\prime \prime}$ latitud Sur y $51^{\circ} 12^{\prime} 32.28^{\prime \prime}$ longitud oeste; con clima de tropical humedo pre montaña; con temperatura promedio de $20^{\circ} \mathrm{C}$, que varía de 16 a $27^{\circ} \mathrm{C}$ y precipitación anual de $1588 \mathrm{~mm}$.

Se utilizaron 96 lechones de linaje Agroceres $x$ PenArlan (48 machos castrados y 48 hembras) destetados a los 21 días de edad y con peso promedio de $6.06 \pm 0.852 \mathrm{~kg}$, los cuales fueron alojados en 48 jaulas (24 jaulas metálicas de piso elevado y 24 en piso concreto) ubicando un macho y una hembra por jaula. Los animales recibieron agua y alimento "ad libitum" durante todo el período experimental.

Tratamientos. Las dietas experimentales fueron formuladas para atender las recomendaciones descritas por Rostagno (15) para cerdos en la fase pre-inicial I ( 1 a 15 días) e inicial II (15 a 30 días). La composición centesimal y nutricional se encuentra en la tabla 1.

Las dietas experimentales tuvieron diferentes inclusiones $(0,1.0,1.5$ y $2.0 \%)$ de L-glutamina (Ajinomoto Brasil) y asociados o no a un desafío 
Table 1. Calculated percentage composition of the basal diet.

\begin{tabular}{lcc}
\hline \multicolumn{1}{c}{ Ingredients \% } & $\begin{array}{c}\text { Pre-initial I } \\
\text { (1-15 days) }\end{array}$ & $\begin{array}{c}\text { Pre-initial II } \\
\text { (15 to 30 days) }\end{array}$ \\
\hline Corn & 53.26 & 67.78 \\
Soybean meal, 45\% & 23.73 & 20.88 \\
Powder Skim Milk & 10.00 & 4.00 \\
Soybean Oil & 3.79 & 0.23 \\
Sugar & 3.00 & 2.00 \\
Whey Powder & 2.00 & 2.00 \\
Dicalcium Phosphate & 1.66 & 1.36 \\
Calcium carbonate & 0.64 & 0.70 \\
L-Lysine HCL & 0.63 & 0.33 \\
Common Salt & 0.51 & 0.36 \\
L-Threonine & 0.29 & 0.09 \\
DL-Methionine & 0.24 & 0.05 \\
Vitini- Sui & 0.20 & 0.20 \\
L-Tryptophan & 0.05 & 0.01 \\
\hline Total & 100.00 & 100.00 \\
\hline Calculated Values & & \\
\hline Metabolizable energy (kcal/kg) & 3.400 & 3.230 \\
Crude protein (\%) & 20.00 & 17.50 \\
Calcium (\%) & 0.85 & 0.72 \\
Available Phospohorus (\%) & 0.50 & 0.39 \\
Digestible Lysine (\%) & 1.45 & 1.02 \\
Digestible Methionine (\%) & 0.55 & 0.32 \\
Digestible Met+Cis (\%) & 0.81 & 0.57 \\
\hline
\end{tabular}

${ }^{1}$ Vitamin-mineral supplement, composition by $\mathrm{kg}$ of product: Vit $\mathrm{A}$, 1.800 .000 UI; Vit D3, 360.000 UI; Vit E, 4.000 UI. Vit K3, 600mg; Vit B1, 280mg; Vit B2, 800mg; Vit B6, 300mg; Vit B12, 3.600mcg; Niacin, $6.000 \mathrm{mg}$; Ac. Pantothenic, $3.200 \mathrm{mg}$; Biotin, 20mg; Folic Ac., $80 \mathrm{mg}$; Choline, 31g; Iron, $20.000 \mathrm{mg}$; Copper, 50.000mg; Cobalt, 120mg; Manganese, $11.000 \mathrm{mg}$; Zinc, $18.000 \mathrm{mg}$; Selenium, 60mg; Iodine, $200 \mathrm{mg}$; Lysine,140g; Antioxidant, $20 \mathrm{~g}$; vehicle q.s.p., $1.000 \mathrm{~g}$.

$\mathrm{mL}$ of skim milk and administered orally between 7:30 and 8:00 $\mathrm{h}$. The other animals also received $5 \mathrm{~mL}$ of skim milk free of LPS orally.

Sampling. Weekly growth performance, feed intake, weight gain and feed conversion of the animals were estimated. For the cortisol and leukogram analysis, blood samples were collected from all animals by puncturing the cranial cava vein. The sample was collected in tubes containing $0.1 \mathrm{~mL}$ anticoagulant EDTA $10 \%$. The first sample was taken on the 43rd day of life between 7:30 and 8:00 h, before LPS inoculation and after an 8-hour fast. Other samples were taken at the same time, day $45^{\circ}$, $47^{\circ}$ and $49^{\circ}$ of life. Leukogram was determined by the method of impedance in hematology BC-280Vet (Mindray) in an authorized clinical veterinary laboratory.

For determination of serum cortisol, samples were collected in tubes without anticoagulant, then were centrifuged at $2000 \mathrm{rpm}$ for 10 minutes. The serum obtained was then transferred to 1.5 $\mathrm{ml}$ micro tubes, identified and stored at $-20^{\circ} \mathrm{C}$ for later analysis. Cortisol was determined by the ELISA technique (Enzyme Linked Immunosorbent Assay) competitive in the immunology IV laboratory at the State University of Londrina. imunológico con LPS de E. coli (serotipo 0111:B4, Sigma Aldrich $\left.{ }^{\circledR}\right)$. El programa de desafío con LPS fue realizado entre el 43 a $47^{\circ}$ día de vida, donde la mitad de los animales recibieron $0.3 \mu \mathrm{g}$ de LPS (serotipo 0111:B4, Sigma Aldrich ${ }^{\circledR}$ ) diluido en $5 \mathrm{~mL}$ de leche desnatada y administrado vía oral, entre las 7:30 y 8:00h. Asimismo, los demás animales recibieron oralmente $5 \mathrm{~mL}$ de leche desnatada libre de LPS.

Toma de muestras. Semanalmente se estimaron los parámetros productivos, consumo de ración, la ganancia de peso y conversión alimenticia de los animales. Para el análisis del cortisol y del leucograma se colectaron muestras de sangre de todos los animales, a través de la punción de la vena cava craneal. La muestra fue colectada en tubos que contenían $0.1 \mathrm{~mL}$ de anticoagulante EDTA $10 \%$. La primera toma fue realizada al día $43^{\circ}$ de vida entre las 7:30 y 8:00h, antes de la inoculación de los LPS y después de un ayuno previo de 8 horas. Las demás muestras fueron realizadas en el mismo horario los día $45^{\circ}, 47^{\circ}$ y $49^{\circ}$ de vida. El leucograma fue determinado por el método de impedancia en hematología BC-280Vet (Mindray) en un laboratorio clínicoveterinario autorizado.

Para la determinación del cortisol sérico las muestras se colectaron en tubos sin anticoagulante, después fueron centrifugadas a $2000 \mathrm{rpm}$ por 10 minutos. El suero obtenido fue transferido a micro tubos de $1.5 \mathrm{~mL}$, identificados y conservados $\mathrm{a}-20^{\circ} \mathrm{C}$ para su posterior análisis. El cortisol fue determinado por la técnica de ELISA (Enzyme Linked Immunosorbent Assay) competitiva en el laboratorio de inmunología IV de la Universidad Estadual de Londrina.

Diseño experimental. Se utilizó un diseño experimental en bloques completos al azar con un arreglo factorial $4 \times 2$ (cuatro niveles de L-glutamina y el desafío inmunológico con o sin LPS de $E$. coli). Los datos obtenidos fueron sometidos a análisis de normalidad, aditividad y homocedasticidad y posteriormente sometidos a análisis de variancia y regresión utilizando el paquete estadístico ExpDes del programa estadístico $\mathrm{R}$ (16). Para el consumo de ración, conversión alimenticia y ganancia de peso, la jaula fue considerada como unidad experimental, siendo dos animales por unidad. Para las demás variables el animal fue considerado unidad experimental.

\section{RESULTADOS}

El consumo diário de alimento y la conversión alimenticia no fueron influenciados por la inclusión 
Experimental design. An experimental design was used on randomized complete blocks with a factorial arrangement $4 \times 2$ (four levels of L-glutamine and immune challenge with or without LPS of E. coli). The data obtained was subjected to analyses of normality, additivity and homoscedasticity and then subjected to an analysis of variance and regression using the statistical package ExpDes from the R statistical program (16). For feed intake, feed conversion and weight gain, the cage was considered as an experimental unit, with two animals per unit. For all other variables the animal was considered the experimental unit.

\section{RESULTS}

Daily feed intake and feed: gain rate conversion were not influenced by the inclusion of L-glutamine or by the $E$. coli LPS immunological challenge. Meanwhile, the immunological challenge influenced weight gain (Table 2 ).

Table 2. Production parameters of weaned piglets supplemented with different levels of glutamine and immunologically challenged with E. coli LPS.

\begin{tabular}{cccc}
\hline \% of Glutamine & DFI (kg) & DWG (kg) & FGR \\
\hline 0 & $0.363 \pm 0.0125$ & $0.203 \pm 0.0132$ & $1.828 \pm 0.1351$ \\
1.0 & $0.382 \pm 0.0231$ & $0.225 \pm 0.0097$ & $1.704 \pm 0.1134$ \\
1.5 & $0.355 \pm 0.0223$ & $0.194 \pm 0.0136$ & $1.864 \pm 0.0864$ \\
2.0 & $0.352 \pm 0.0165$ & $0.216 \pm 0.0094$ & $1.612 \pm 0.0768$ \\
\hline LPS, $\mu \mathrm{g}$ & & & \\
\hline 0.0 & $0.371 \pm 0.0130$ & $0.221 \pm 0.0089 \mathrm{a}$ & $1.711 \pm 0.0716$ \\
0.3 & $0.355 \pm 0.0137$ & $0.198 \pm 0.0072 \mathrm{~b}$ & $1.793 \pm 0.0791$ \\
\hline General Average & $0.363 \pm 0.0094$ & $0.210 \pm 0.0059$ & $1.752 \pm 0.0531$ \\
\hline CV(\%) & 17.98 & 19.51 & 21.00 \\
\hline
\end{tabular}

Table 3. Interaction between glutamine and LPS levels for variables daily weight gain (DWG) and feed conversion (FGT).

\begin{tabular}{|c|c|c|c|c|}
\hline \multirow[b]{2}{*}{$\mathrm{Gln}, \%^{1}$} & \multicolumn{2}{|c|}{ DWG (kg) LPS } & \multicolumn{2}{|c|}{ FGR LPS } \\
\hline & $0.3 \mu \mathrm{g}$ & $0.0 \mu \mathrm{g}$ & $0.3 \mu \mathrm{g}$ & $0.0 \mu \mathrm{g}$ \\
\hline 0 & $\begin{array}{c}0.17 \\
\pm 0.009^{b}\end{array}$ & $\begin{array}{c}0.24 \\
\pm 0.010^{\mathrm{a}}\end{array}$ & $\begin{array}{c}2.13 \\
\pm 0.207^{\mathrm{a}}\end{array}$ & $\begin{array}{c}1.53 \\
\pm 0.061^{\mathrm{b}}\end{array}$ \\
\hline 1.0 & $\begin{array}{c}0.21 \\
\pm 0.012^{\mathrm{a}}\end{array}$ & $\begin{array}{c}0.24 \\
\pm 0.010^{\mathrm{a}}\end{array}$ & $\begin{array}{c}1.70 \\
\pm 0.171^{\mathrm{a}}\end{array}$ & $\begin{array}{c}1.70 \\
\pm 0.164^{\mathrm{a}}\end{array}$ \\
\hline 1.5 & $\begin{array}{c}0.21 \\
\pm 0.017^{\mathrm{a}}\end{array}$ & $\begin{array}{c}0.17 \\
\pm 0.018^{\mathrm{b}}\end{array}$ & $\begin{array}{c}1.75 \\
\pm 0.081^{\text {a }}\end{array}$ & $\begin{array}{l}1.97 \\
\pm 0.145^{\mathrm{a}}\end{array}$ \\
\hline 2.0 & $\begin{array}{c}0.20 \\
\pm 0.010^{\mathrm{a}}\end{array}$ & $\begin{array}{c}0.23 \\
\pm 0.013^{\mathrm{a}}\end{array}$ & $\begin{array}{c}1.58 \\
\pm 0.067^{\mathrm{a}}\end{array}$ & $\begin{array}{c}1.64 \\
\pm 0.141^{\text {a }}\end{array}$ \\
\hline \multirow[t]{2}{*}{$\mathrm{p}$-value } & 0.0618 & 0.0021 & 0.0317 & 0.1754 \\
\hline & & \multicolumn{3}{|c|}{ Regression Equation } \\
\hline GDP $(\mathrm{kg})$ & $0.0 \mu \mathrm{g}$ & $\hat{Y}=0.2413$ & $\begin{array}{l}69 X-0.498 \\
\left(R^{2}=1.00\right)\end{array}$ & $+0.1665 \times 3$ \\
\hline
\end{tabular}

$\frac{\mathrm{CA} \quad 0.3 \mu \mathrm{g} \quad \hat{\mathrm{Y}}=2.080-0.254 \mathrm{X}\left(\mathrm{R}^{2}=0.762\right)}{{ }^{1} \text { Values followed by different letters in the row differ statistically by the }}$ $F$ test $(p<0.05)$. de L-glutamina ni por el desafio inmunológico de LPS de E. coli. Entre tanto, el desafio inmunológico influyó en la ganancia de peso (Tabla 2).

Se observó interacción entre los niveles de glutamina y el desafío con LPS para la ganancia de peso y conversión alimenticia (Tabla 3). Los animales no desafiados presentaron un efecto cúbico de los niveles de glutamina sobre la ganancia de peso, siendo el punto máximo $0.41 \%$ de inclusión de L-glutamina.

Además, no se observaron diferencias en la ganancia diaria de peso entre los animales desafiados o no suplementados con 1.0 y $2.0 \%$ de glutamina (Tabla 3 ).

Para los animales que no fueron suplementados con L-glutamina y no fueron desafiados se observó una mayor ganancia diaria de peso. Sin embargo, para los animales suplementados con $1.5 \%$ de glutamina y desafiados obtuvieron una ganancia de peso mayor con relación a los no desafiados (Tabla 3).

La conversión alimenticia de los animales desafiados presentó un efecto lineal decreciente a medida que aumentaba la inclusión de L-glutamina, indicando una reducción de 0.254 kg para cada nivel de inclusión (Tabla 3). Los animales que no fueron suplementados con L-glutamina presentaron diferencia $(p<0.05)$ en la conversión alimentar.

La concentración de cortisol sérico no fue influenciada por el desafío inmunológico ni por la inclusión de L-glutamina (Tabla 4).

Entretanto, hubo interacción entre los niveles de glutamina y el desafío con LPS para los valores de cortisol (Tabla 5). Los animales no desafiados presentaron un efecto cuadrático, obteniendo un valor máximo de cortisol con la inclusión de $1.45 \%$ de L-glutamina. Los animales desafiados presentaron un efecto cúbico siendo el valor máximo para el cortisol con un nivel de $1.68 \%$ y un valor mínimo de $0.51 \%$ de inclusión de L-glutamina.

También, se presentó diferencia entre los niveles de glutamina $0,1.0$ y 1.5 para los animales desafiados inmunológicamente o no. Entre tanto, los niveles de cortisol de los animales desafiados o no y suplementados con $2 \%$ de L-glutamina se mantuvieron similares durante el período experimental (Tabla 5).

El conteo de leucocitos y monocitos no fue influenciado por los niveles de L-glutamina, entre tanto, se presentó un efecto cuadrático en los eosinófilos y linfocitos influenciados por los diferentes niveles de L-glutamina; siendo el valor máximo de $1.30 \%$ y 
Interaction between levels of glutamine and the LPS challenge were observed for weight gain and feed conversion (Table 3 ). Unchallenged animals had a cubic effect of glutamine levels on weight gain, the maximum point of inclusion of L-glutamine being $0.41 \%$.

In addition, there were no observed differences in daily weight gain between challenged or unchallenged animals supplemented with 1.0 and $2.0 \%$ glutamine (Table 3 ).

For animals that were not supplemented with L-glutamine and were not challenged, increased daily weight gain was observed. However, challenged animals supplemented with $1.5 \%$ glutamine obtained a greater weight gain compared to non-challenged (Table 3 ).

Feed conversion of the challenged animals showed a decreasing linear effect with increasing inclusion of L-glutamine, indicating a reduction of 0.254 $\mathrm{kg}$ for each inclusion level (Table 3 ). The animals that were not supplemented with L-glutamine showed a $(p<0.05)$ difference in feed conversion.

Serum cortisol concentration was not influenced by the immunological challenge or by the inclusion of L-glutamine (Table 4).

Meanwhile, there was interaction between levels of glutamine and the LPS challenge for cortisol values (Table 5). Unchallenged animals showed a quadratic effect, obtaining a maximum value of cortisol with the inclusion of $1.45 \%$ of L-glutamine. The challenged animals showed a cubic effect reaching a maximum cortisol value with a level of $1.68 \%$ of L-glutamine inclusion, and a minimum value with $0.51 \%$ of L-glutamine inclusion.

There was also a difference between glutamine levels $0,1.0$ and 1.5 for immunologically challenged animals or unchallenged animals.

Table 4. Serum cortisol values $(\mathrm{ng} / \mathrm{mL})$ of weaned piglets immunologically challenged with $E$. coli LPS and supplemented with L-glutamine.

\begin{tabular}{cc}
\hline Levels of Glutamine, $\%$ & Serum Cortisol, $\mathbf{n g} / \mathbf{m L}$ \\
\hline 0 & $66.18 \pm 6.98$ \\
1.0 & $92.20 \pm 7.30$ \\
1.5 & $114.86 \pm 5.60$ \\
2.0 & $106.38 \pm 6.92$ \\
\hline \multicolumn{2}{|c}{} \\
\hline $0.3 \mu \mathrm{g}$ & $89.80 \pm 5.75$ \\
General average & $101.29 \pm 4.27$ \\
$\mathrm{CV}(\%)$ & $95.51 \pm 3.60$ \\
& 51.08 \\
\hline
\end{tabular}

0.59\% de inclusión, respectivamente. Los neutrófilos presentaron un efecto lineal creciente con cada nivel de inclusión de la L-glutamina (Tabla 6).

El desafío inmunológico influyó sobre el conteo de leucocitos; presentando valores superiores para los animales desafiados con LPS de E. coli (Tabla 6).

Para los leucocitos hubo interacción entre los niveles de L-glutamina y el desafío inmunológico (Tabla 7).

Hubo diferencia entre los animales suplementados con $0,1.0$ y $1.5 \%$ de L-glutamina y desafiados inmunológicamente, entre tanto, los animales desafiados y suplementados con L-glutamina $(1.0,1.5$ y $2.0 \%$ ) presentaron un mayor conteo de linfocitos, comparados con los no desafiados; sin embargo, esta diferencia se invirtió al suplementar los lechones con $1.5 \%$ de L-glutamina (Tabla 7).

\section{DISCUSIóN}

El consumo de alimento es uno de los factores que tienen gran importancia sobre el desarrollo del tracto digestivo de lechones recién destetados. La producción enzimática es proporcional a la cantidad del sustrato en el tracto intestinal, así, el efecto observado sobre la ganancia de peso con la inclusión de $1.5 \%$ de L-glutamina se debe principalmente al aumento en la actividad de la amilasa y de la tripsina pancreática de los lechones, lo cual mejora la digestibilidad (17).

El efecto positivo sobre la conversión alimenticia puede ser explicado por la acción de la glutamina sobre el metabolismo, estructura y función intestinal, el cual inhibe la disminución del crecimiento y la atrofia de las vellosidades de la mucosa de los animales desafiados con LPS, aumenta la absorción de los nutrientes de la dieta y mejora la eficiencia alimenticia (18).

Table 5. Interaction between levels of glutamine and LPS for the serum cortisol.

\begin{tabular}{|c|c|c|}
\hline & \multicolumn{2}{|c|}{ Cortisol sérico, $\mathrm{ng} / \mathrm{mL}$} \\
\hline $\mathrm{G} \ln ^{1 \%}$ & $0.3 \mu \mathrm{g}$ & $0.0 \mu \mathrm{g}$ \\
\hline 0 & $105.77 \pm 8.88^{\mathrm{a}}$ & $33.48 \pm 3.11^{\mathrm{b}}$ \\
\hline 1.0 & $68.66 \pm 5.02^{\mathrm{b}}$ & $116.81 \pm 11.95^{a}$ \\
\hline 1.5 & $126.71 \pm 7.49^{a}$ & $102.48 \pm 7.68^{\mathrm{b}}$ \\
\hline 2.0 & $104.68 \pm 7.98^{\mathrm{a}}$ & $108.16 \pm 11.55^{\mathrm{a}}$ \\
\hline \multirow[t]{2}{*}{$\mathrm{p}$-value } & 0.00065 & 0.00034 \\
\hline & \multicolumn{2}{|c|}{ Regression Equation } \\
\hline \multirow{2}{*}{ Serum Cortisol } & \multicolumn{2}{|c|}{$\begin{array}{c}\hat{Y}=105.7695-335.9840 x+430.0320 x^{2}-131.1558 x^{3} \\
\left(R^{2}=1.00\right)\end{array}$} \\
\hline & \multicolumn{2}{|c|}{$\begin{array}{c}\hat{Y}=34.9786+110,4813 x-38.0710 x^{2} \\
\left(R^{2}=0.9407\right)\end{array}$} \\
\hline
\end{tabular}
row differ statistically by the $F$ test $(p<0.05)$. 
Meanwhile, cortisol levels in challenged and unchallenged animals supplemented with $2 \%$ L-glutamine remained similar throughout the experimental period (Table 5).

The counting of leukocytes and monocytes was not influenced by levels of L-glutamine, meanwhile, there was a quadratic effect on eosinophils and lymphocytes influenced by different levels of L-glutamine; with a maximum value of $1.30 \%$ and $0.59 \%$ of inclusion, respectively. Neutrophils showed an increasing linear effect with each level of L-glutamine inclusion (Table 6).

The immunological challenge influenced the leukocyte count; with higher values for the animals that were challenged with E. coli LPS (Table 6).

For leukocytes, there was interaction between L-glutamine levels and the immunological challenge (Table 7).

There was a difference between the animals supplemented with $0,1.0$ and $1.5 \%$ of L-glutamine and the immunologically challenged, meanwhile, challenged animals supplemented with L-glutamine $(1.0,1.5$ and $2.0 \%)$ had increased lymphocyte counts compared with unchallenged; however, this difference was reversed by supplementing piglets with $1.5 \%$ of L-glutamine (Table 7).

\section{DISCUSSION}

Food consumption is one of the factors that have great importance on the development of the digestive tract of recently weaned piglets. Enzyme production is proportional to the amount of substrate in the intestinal tract, therefore, the observed effect on weight gain with the inclusion of $1.5 \%$ of L-glutamine is largely due to the increase in activity of amylase and pancreatic trypsin in the piglets, improving digestibility (17).

The positive effect on feed conversion can be explained by glutamine action on the metabolism, intestinal structure and function, which inhibits the decrease of growth and atrophy of the mucosal villi in animals challenged with LPS, increases absorption of dietary nutrients and improves feed efficiency (18).

The differences obtained in feed conversion for animals that didn't receive inclusion of L-glutamine (which were not challenged with E. coli LPS) may be due to metabolic stress processes during which there is a reduction in luminal transport of glutamine and in the activity
Table 6. Defense cell values for weaned pigs fed different levels of L-glutamine and immunologically challenged with $E$. coli LPS.

Gln ${ }^{1} \%$ Leukocytes Eosinophils Monocytes Neutrophils Lymphocytes

\begin{tabular}{|c|c|c|c|c|c|}
\hline 0 & $\begin{array}{c}15847.9 \\
\pm 527.1\end{array}$ & $\begin{array}{c}1.51 \\
\pm 0.186\end{array}$ & $\begin{array}{c}1.58 \\
\pm 0.204\end{array}$ & $\begin{array}{c}41.71 \\
\pm 1.560\end{array}$ & $\begin{array}{c}55.04 \\
\pm 1.544\end{array}$ \\
\hline 1.0 & $\begin{array}{c}16192.7 \\
\pm 408.5\end{array}$ & $\begin{array}{c}0.97 \\
\pm 0.158\end{array}$ & $\begin{array}{c}1.97 \\
\pm 0.267\end{array}$ & $\begin{array}{c}41.20 \\
\pm 1.293\end{array}$ & $\begin{array}{c}55.75 \\
\pm 1.270\end{array}$ \\
\hline 1.5 & $\begin{array}{c}17145.8 \\
\pm 590.6\end{array}$ & $\begin{array}{c}0.89 \\
\pm 0.141\end{array}$ & $\begin{array}{c}1.51 \\
\pm 0.214\end{array}$ & $\begin{array}{c}44.57 \\
\pm 1.386\end{array}$ & $\begin{array}{c}52.94 \\
\pm 1.448\end{array}$ \\
\hline 2.0 & $\begin{array}{c}18118.5 \\
\pm 769.4\end{array}$ & $\begin{array}{c}1.10 \\
\pm 0.158\end{array}$ & $\begin{array}{c}2.10 \\
\pm 0.247\end{array}$ & $\begin{array}{c}47.28 \\
\pm 1.783\end{array}$ & $\begin{array}{l}46.91 \\
\pm 1.75\end{array}$ \\
\hline $\mathrm{p}$-value & 0.326 & $0.041^{2}$ & 0.184 & $0.006^{3}$ & 0.00002 \\
\hline \multicolumn{6}{|c|}{ LPS } \\
\hline $0.0 \mu \mathrm{g}$ & $\begin{array}{l}16197.4 \\
\pm 464.4 \mathrm{~b}\end{array}$ & $\begin{array}{c}1.20 \\
\pm 0.12\end{array}$ & $\begin{array}{c}1.74 \\
\pm 0.16\end{array}$ & $\begin{array}{l}43.41 \\
\pm 1.07\end{array}$ & $\begin{array}{l}52.23 \\
\pm 1.11\end{array}$ \\
\hline $0.3 \mu \mathrm{g}$ & $\begin{array}{l}17496.3 \\
\pm 367.4 a\end{array}$ & $\begin{array}{c}1.00 \\
\pm 0.10\end{array}$ & $\begin{array}{c}1.85 \\
\pm 0.17\end{array}$ & $\begin{array}{l}44.07 \\
\pm 1.09\end{array}$ & $\begin{array}{l}52.97 \\
\pm 1.08\end{array}$ \\
\hline $\begin{array}{l}\text { General } \\
\text { Average }\end{array}$ & $\begin{array}{c}16855.0 \\
\pm 297.4\end{array}$ & $\begin{array}{c}1.10 \\
\pm 0.08\end{array}$ & $\begin{array}{c}1.79 \\
\pm 0.11\end{array}$ & $\begin{array}{l}43.74 \\
\pm 0.76\end{array}$ & $\begin{array}{l}52.60 \\
\pm 0.77\end{array}$ \\
\hline$C V, \%$ & 31.56 & 131.27 & 117.52 & 31.30 & 26.39 \\
\hline
\end{tabular}

${ }^{1}$ Levels of L-glutamine inclusion. ${ }^{2} \hat{Y}=1.512-0.915 x+0.350 x^{2}\left(R^{2}=\right.$ $0.99) ;{ }^{3} \hat{Y}=40.462+2.828 x\left(R^{2}=0.61\right) ;{ }^{4} \hat{Y}=55.006+5.843 x-4.921 x^{2}$ $\left(R^{2}=0.91\right)$. Values followed by different letters in the column differ statistically by the $F$ test $(p<0.05)$.

Table 7. Interaction between levels of glutamine and LPS for the Leukocyte variable.

\begin{tabular}{ccc}
\hline \multirow{2}{*}{ Gln $^{\mathbf{1} \%}$} & \multicolumn{2}{c}{ Leukocytes } \\
\cline { 2 - 3 } & \multicolumn{2}{c}{$\mathbf{0 . 3 \mu \mathbf { \mu g }}$} \\
\hline 0 & $17702.86 \pm 768.6934 \mathrm{~g}$ & $14044.44 \pm 606.4233 \mathrm{~b}$ \\
1.0 & $17334.88 \pm 649.6234 \mathrm{a}$ & $14965.00 \pm 415.5094 \mathrm{~b}$ \\
1.5 & $16013.95 \pm 535.2634 \mathrm{~b}$ & $18362.00 \pm 1041.5945 \mathrm{a}$ \\
2.0 & $19082.50 \pm 900.1488 \mathrm{a}$ & $17178.05 \pm 1237.7677 \mathrm{a}$ \\
p-valor & 0.0686 & 0.0008 \\
\hline \multicolumn{3}{c}{ Regression Equation } \\
\hline Leukocyte & $0.0 \mu \mathrm{g}$ & $\hat{\mathrm{Y}}=13929.907+1975.748 \times(\mathrm{R} 2=0.6993)$ \\
\hline
\end{tabular}

${ }^{1}$ Levels of L-glutamine inclusion. Values followed by different letters in the line differ statistically by the $F$ test $(p<0.05)$.

Las diferencias obtenidas en la conversión alimenticia para los animales que no recibieron inclusión de L-glutamina (que fueron o no desafiados con LPS de $E$. coli), pueden ser debidas a que durante los procesos de estrés metabólico ocurre una reducción en el transporte luminal de la glutamina y de la actividad de la glutaminasa de la mucosa (19), existiendo la posibilidad de que el intestino supla las necesidades de otros órganos como parte del proceso para priorizar la síntesis proteica y de esa manera no sea comprometido el aprovechamiento de los nutrientes. 
of the mucosal glutaminase (19), with the possibility of the intestine meeting the needs of other organs as part of the process for prioritizing protein synthesis and thus not compromising the use of nutrients.

Stress is a physical-chemical or emotional process which promotes the release of pro-inflammatory cytokines, corticotropin-releasing hormone and cortisol. When stressors become chronic they cause cortisol levels to remain elevated, which leads to an imbalance of neuro-endocrine immunological interactions (20). Exposure to low doses of E. coli LPS causes activation of the hypothalamic-pituitaryadrenal axis, increasing cortisol levels in the blood, and indicates the level of stress to which the animal is being subjected $(19,21)$.

Using $0.3 \mu \mathrm{g}$ of LPS/pig/day did not follow the physiological pattern of moderate or transient cortisol during collections, as the values were expected to decrease in comparison to the first challenge. Increased cortisol values in challenged animals can be explained by the effects of endotoxemia induced by $E$. coli LPS and the effect of stress (20). Glutamine may reduce the synthesis of cortisol in the adrenal cortex due to reduction in key enzyme activities or in the availability of NADPH. In normal situations, cortisol values may vary, it is therefore necessary to consider normal periodic changes and physiological responses to stress (21).

The observed increase in the number of lymphocytes in challenged animals is attributed to the role of glutamine as a source of ATP for lymphocytes and macrophages, enhancing the immune response of the performance of the intestinal barrier (22). In addition, a simple stress stimulus causes changes in the number of leukocytes in the blood, which can affect the immune system's availability in responding to these changes.

The difference observed in leukocytes for challenged or unchallenged animals indicates that LPS stimulates increased production of leukocytes and this increase is dependent on extracellular glutamine present at the time of challenge; however, these concentrations may also vary depending on genetics, environment, the health of the animals and serum cortisol values (23).

The evident effect for neutrophils was possibly caused by the inclusion of L-glutamine on the immune response, as the amino acid enhances proliferation of phagocytic activity and the rate of production of superoxide (free radical necessary for bacterial death), decreasing the number of $E$. coli present after a challenge (24).
El estrés es un proceso físico-químico o emocional que promueve la liberación de citocinas proinflamatorias, hormona liberadora de corticotropina y cortisol. Cuando los estímulos estresantes se vuelven crónicos hacen que los niveles de cortisol se mantengan elevados, lo que induce a un desequilibrio de las interacciones neuroendocrinoinmunológicas (20). La exposición a bajas dosis de LPS de $E$. coli causa la activación del eje hipotálamico-hipofisiario-adrenal, aumentando los niveles de cortisol en la sangre, e indica el nivel de estrés a que el animal está siendo sometido $(19,21)$.

La utilización de $0.3 \mu \mathrm{g}$ de LPS/lechón/día no siguió un patrón fisiológico de cortisol moderado o transitorio durante las colectas, una vez que se esperaban que los valores disminuyeran en comparación al primer desafío. El aumento en los valores del cortisol de los animales desafiados puede ser explicado por los efectos de la endotoxemia inducida por el LPS de $E$. coli y los efectos del estrés (20). La glutamina puede reducir la síntesis del cortisol en la corteza adrenal debido a la reducción en las actividades de las enzimas claves o en la disponibilidad del NADPH. En situaciones normales, los valores del cortisol pueden variar, por tanto, es necesario considerar las variaciones periódicas normales y las respuestas fisiológicas al estrés (21).

El aumento observado en el número de linfocitos de los animales desafiados es atribuido a la función de la glutamina como fuente de ATP para los linfocitos y macrófagos mejorando la respuesta inmunológica y la función de la barrera intestinal (22). Además, un simple estímulo al estrés genera cambios en el número de leucocitos en sangre, los cuales pueden afectar la disponibilidad del sistema inmune en responder a estas alteraciones.

La diferencia observada para los leucocitos en los animales desafiados o no indican que el LPS estimula la mayor producción de leucocitos y que este aumento depende de la glutamina extracelular presente en el momento del desafío; sin embargo, estas concentraciones también pueden variar en función de la genética, el ambiente, el estado de salud de los animales y de los valores de cortisol séricos (23).

El efecto evidenciado para los neutrofilos posiblemente fue causado por la inclusión de la L-glutamina sobre la respuesta inmunitaria, una vez que este aminoácido mejora la proliferación de la actividad fagocítica y de la tasa de producción de superóxido (radical libre necesario para la muerte bacteriana), disminuyendo el número de $E$. coli presente después de un desafío (24).

Cuando los animales son desafiados con LPS de E. coli y son suplementados con L-glutamina, se 
When animals are challenged with $E$. coli LPS and are supplemented with L-glutamine, one can observe the beneficial effect of the amino acid, attenuating the decrease in growth and inefficient use of nutrients. In this case, L-glutamine serves as a metabolic regulator to increase protein synthesis and reduce protein catabolism in infectious and inflammatory processes, maintaining a rate of protein deposition in the skeletal muscle (25).

Depending on the severity of the challenge, it is difficult to correctly estimate the beneficial effect of L-glutamine inclusion, since inclusion levels determine different responses in the evaluated parameters. Furthermore, it is known that immunological challenges cause inflammatory alterations and a variety of physiological responses (26). When challenged and not supplemented with L-glutamine, the release of cytokines by macrophages occurs, and the activation of the hypothalamic-pituitary axis, which causes protein degradation in skeletal muscle, reducing intracellular concentration of glutamine, altering the intermediary metabolism and absorption of nutrients (27).

In conclusion L-glutamine inclusion of up to $2 \%$ in the diet improves the feed conversion of animals subjected to the challenge with $E$. coli LPS. Therefore, positive responses regarding cellular immunity serum in weaned piglets are due to the inclusion of L-glutamine, and its use is recommended at this stage.

\section{Acknowledgements}

To the Foundation for Research Support of Mato Grosso- Brazil (FAPEMAT) for the research grant, and to Ajinomoto Brazil / Animal Nutrition for donating L-glutamine and to CAPES for the PEC-PG program scholarship. puede observar el efecto benéfico del aminoácido, atenuando la disminución en el crecimiento y la utilización ineficiente de los nutrientes. En este caso la L-glutamina actúa como un regulador metabólico para aumentar la síntesis proteica y disminuir el catabolismo proteico en procesos infecciosos e inflamatorios, manteniendo una tasa de deposición de proteína en el músculo esquelético (25).

Dependiendo de la intensidad del desafío es difícil estimar correctamente el efecto benéfico de la inclusión de la L-glutamina, ya que los niveles de inclusión determinan las diferentes respuestas en los parámetros evaluados. Además, es conocido que los desafíos inmunológicos causan alteraciones inflamatorias y una variedad de respuestas fisiológicas (26). Cuando se desafia y no se suplementa con L-glutamina, ocurre la liberación de citocinas por los macrófagos y una activación del eje hipotálamo-hipófisis, que causa degradación de la proteína en el músculo esquelético, reduciendo la concentración intracelular de la glutamina, alterando el metabolismo intermediario, así como la absorción de nutrientes (27).

En conclusión la inclusión de L-glutamina hasta un $2 \%$ en la dieta mejora la conversión alimenticia de los animales sometidos al desafío con LPS de $E$. coli. Por tanto, las respuestas positivas relacionadas con la inmunidad celular sérica en lechones destetados se debe a la inclusión de la L-glutamina, recomendándose su uso en esta fase.

\section{Agradecimientos}

A la Fundación de Amparo a la Investigación de Mato Grosso- Brasil (FAPEMAT) por la beca de investigación, así como a Ajinomoto Brasil/ Animal Nutrition por la donación de la L-glutamina utilizada y a CAPES por la beca de estudio del programa PEC-PG.

\section{REFERENCES}

1. Lallès JP, Boudry G, Favier C, Le Floc'h N, Luron I, Montagne $L$, et al. Gut function and dysfunction in young pigs: physiology. Anim Res 2004; 53:301-316.

2. Reis-de Souza TC, Mariscal-Landín G, EscobarGarcía K, Aguilera-Barreyro A, Magné-Barrón A. Cambios nutrimentales en el lechón y desarrollo morfofisiológico de su aparato digestivo. Vet Mex 2012; 43(2): 155-173.

3. Pluske JR, Kerton DK, Cranwell PD, Campbell C, Mullan BP, King $\mathrm{RH}$, et al. Age, sex, and weight at weaning influence organ weight and gastrointestinal development of weanling pigs. Aust J Agr Res 2003; 54(5):515-527.
4. Silva Da LP, Nornberg JL. Prebíóticos na nutrição de não ruminantes. Cienc Rural 2003;33:983990.

5. Moeser AJ, Klok CV, Ryan KA, Wooten JG, Little $D$, Cook $V L$, et al. Stress signaling pathways activated by weaning mediate intestinal dysfunction in the pig. Am J Physiol Gastrointest Liver Physiol 2007; 292(1):173-181.

6. Rhoads JM, Wu G. Glutamine, arginine, and leucine signaling in the intestine. Amino Acids 2009;37(1):111-22. 
7. Wu G, Bazer FW, Johnson GA, Knabe DA, Burghardt RC, Spencer TE, et al. Important roles for L-glutamine in swine nutrition and production. J Anim Sci 2010; Epub.

8. Li XL, Rezaei R, Li P, Wu G. Composition of amino acids in feed ingredients for animal diets. Amino Acids 2011; 40(4):1159-68.

9. Watford M, Kutschenko M, Nogueira ET. Optimal dietary glutamine for growth and development. R Bras Zootec 2011; 40:384-390.

10. Ribeiro $A M L$, Pinheiro $C C$, Gianfelice $M$. Nutrientes que afetam a imunidade dos leitões. Acta sci vet 2008; 36 suppl1:119-124.

11. Zavarize KC, Menten JFM, Traldi AB, Santarosa J, da Silva CLS. Utilização de glutamina na nutrição de monogástricos. Ver port ciênc vet 2010; 105:573-576.

12. Lopes P.F. Efeito da glutamina sobre a parede intestinal e sua aplicabilidade potencial em coloproctologia. Rev bras Coloproctol 2005; 25:75-78.

13. Hou Y, Wang L, Ding B, Liu Y, Zhu H, Liu J, et al. Dietary a-ketoglutarate supplementation ameliorates intestinal injury in lipopolysaccharidechallenged piglets. Amino Acids 2010; 39:555564.

14. Liu Y, Huang J, Hou Y, Zhu H, Zhao S, Ding $B$, et al. Dietary arginine supplementation alleviates intestinal mucosal disruption induced by Escherichia coli lipopolysaccharide in weaned pigs. $\mathrm{Br}$ J Nutr 2008; 100(3):552-60.

15. Rostagno HS. Tabelas Brasileiras para Aves e Suínos. 3.ed. Viçosa-MG, Brasil: Universidade Federal de Viçosa. 2011.

16. R DEVELOPMENT CORE TEAM. R: A language and environment for statistical computing. $R$ Development Core Team. R Foundation for Statistical Computing. 2012

17. Tucci FM, Thomaz MC, Pizauro Júnior JM, Hannas MI, Scandolera AJ, Lemos Budiño FE. Agentes tróficos na dieta de leitões desmamados sobre a atividade das enzimas digestivas e o desempenho. Braz J Vet Res Anim Sci 2011,48(4):289-298.

18. Cabrera RA, Ursy JL, Arellano C, Nogueira $E T$, Kutschenko M, Moeser AJ. Effects of creep feeding and supplemental glutamine or glutamine plus glutamate (Aminogut) on pre- and post-weaning growth performance and intestinal health of piglets. J Anim Sci Biotech 2013; 4(1):29.

19. Curi R, Newsholme P, Procopio J, Lagranha C, Goriao R Pithon-Curi TC. Glutamine gene expression and cell function. Front Biosci 2007; $12: 344-357$.

20. García-Herrera J, Marca MC, Brot-Laroche E, Guillén N, Acin S, Navarro MA et al. Protein kinases, TNF-a, and proteasome contribute in the inhibition of fructose intestinal transport by sepsis in vivo. Am J Physiol Gastrointest Liver Physiol 2008, 294:155-164.

21. Fagundes ACA, Negrão JA, Da Silva RG, Gomes JDF, Souza LDO, Fukushima RS. Environmental temperature and serum cortisol levels in growing-finishing pigs. Braz J Vet Res Anim Sci 2008; 45(supl):136-140.

22. García-de-Lorenzo A , Zarazaga A, García-Luna PP , Gonzalez-Huix F , López-Martínez J ,Miján $A$ et al. Clinical evidence for enteral nutrition support with glutamine: a systematic review. Nutrition 2003; 19(9):805-811.

23. Kick AR, Tompkins MB, Flowers $W L$, Whisnat CS, Almond GW. Effect of stress associated with weaning on the adaptive immune system in pigs. J Anim Sci 2012; 90(2):649-656.

24. Leandro CG, Nascimento E, Azevedo MM, Viegas A, Albuquerque C, Cavalcanti CB et al. Efeito da L-glutamina sobre o perfil leucocitário e a função fagocitica de macrófagos de ratos estressados. Rev Nutr 2006; 19(4):437-444.

25. Yi GF, Carrol JA, Allee GL, Gaines DC, Kendall JK, Ursy $Y$ et al. Effect of glutamine and spraydried plasma on growth performance, small intestinal morphology, and immune responses of Escherichia coli K88+-challenged weaned pigs. J Anim Sci 2005; 83:634-643.

26. Parra JS, Agudelo J, Sanín D, Forero J, Muskus C, Lopez-Herrera A. Intestinal expression of proinflammatory cytokines induced by oral intake of lipopolysaccharide (LPS) from E. coli in weaned pigs. Rev Colomb Cienc Pecu 2013; 26:108-118.

27. Pié S; Lallès JP, Blazy F, Laffite J, Sève B, Oswald IP. Weaning is associated with an up regulation of expression of inflammatory cytokines in the intestine of piglets. J Nutr 2004; 134: 641-647. 